\title{
Matthew Boulton et ses réseaux, à partir des Archives de Soho à Birmingham
}

Matthew Boulton et ses réseaux, à partir des Archives de Soho à Birmingham

Peter M. Jones

Traducteur : Liliane Perez

\section{OpenEdition}

\section{Journals}

Édition électronique

URL : http://journals.openedition.org/dht/466

DOI : $10.4000 /$ dht.466

ISSN : $1775-4194$

Éditeur :

Centre d'histoire des techniques et de l'environnement du Cnam (CDHTE-Cnam), Société des élèves du CDHTE-Cnam

\section{Édition imprimée}

Date de publication : 31 mars 2009

Pagination : 11-18

ISBN : 978-2-95-30779-3-3

ISSN : 0417-8726

\section{Référence électronique}

Peter M. Jones, « Matthew Boulton et ses réseaux, à partir des Archives de Soho à Birmingham »,

Documents pour l'histoire des techniques [En ligne], 17 | Ter $^{\mathrm{e}}$ semestre 2009, mis en ligne le 06 avril 2011, consulté le 08 septembre 2020. URL : http://journals.openedition.org/dht/466 ; DOI : https://doi.org/ $10.4000 /$ dht. 466 


\title{
Matthew Boulton et ses réseaux, à partir des Archives de Soho à Birmingham*
}

\author{
Peter M. Jones \\ Department of Modern History \\ University of Birmingham
}

\begin{abstract}
RÉSUMÉ
Cet article attire l'attention sur le fait que la bibliothèque municipale de la ville de Birmingham conserve l'un des fonds d'archives industrielles les mieux préservés au monde concernant les XVIIIe et XIXe siècles. On y décrit les recherches menées dans ces archives afin de mettre à l'épreuve I'hypothèse avancée par Joel Mokyr dans The gifts of Athena selon laquelle certains sites privilégiés en Europe ont bénéficié des « Lumières industrielles" pendant le dernier tiers du XVIIIle siècle. En se concentrant sur les visiteurs de la manufacture de Soho de Matthew Boulton située aux alentours de Birmingham, l'article souligne également l'importance de la littérature de voyage pour les historiens des sciences et de l'industrie
\end{abstract}

Résumés et mots clés en anglais sont regroupés en fin de volume, accompagnés des mots clés français

L e fabricant de Birmingham et patron des arts, Matthew Boulton (1728 - 1809), reste mal connu des chercheurs travaillant sur le siècle des Lumières, et même de ceux qui nous ont encouragés à nous concentrer sur les " contextes nationaux " ' de l'histoire des savoirs et de leur circulation au siècle des Lumières. Le même constat vaut pour les sources archivistiques qui permettent de considérer la carrière de Boulton comme celle d'un industriel majeur et représentant revendiqué de la civilité des Lumières en Angleterre. Ce riche fonds d'archives, connu sous le nom d'Archives de Soho, est pourtant conservé à la bibliothèque municipale de la ville de Birmingham².

1 Roy Porter et Miklaus Teich éd., The Enlightenment in national context, Cambridge, Cambridge University Press, 1981 ; Roy Porter, Enlightenment : Britain and the creation of the modern world, London, Allen Lane, 2000.

2 Birmingham Central Library, Floor 6, Archives and Heritage Service, Chamberlain Square, Birmingham, B3 $3 H Q$, Royaume Uni.
L'enjeu de cet article est de rappeler qui est Matthew Boulton et d'attirer l'attention sur ces collections, plus particulièrement sur le potentiel qu'elles recèlent pour les chercheurs. Dans la deuxième partie du texte, j'expose mes recherches en cours dans ces archives sur l'économie de la connaissance en Europe au XVIII siècle à partir de perspectives concernant l'histoire des sciences et des techniques. Puisque nous formons un groupe éclectique de chercheurs intéressés par le XVIIII siècle à des multiples égards, cet article est aussi l'occasion de mentionner brièvement plusieurs autres pistes d'enquête que peuvent offrir ces archives.

Mon propre intérêt pour Matthew Boulton découle de son statut d'entrepreneur archétypal des Lumières provinciales anglaises ${ }^{3}$. Mais cet intérêt n'aurait pas été soutenu sans la connaissance extraordinairement

3 Depuis le Congrès des Lumières (2007), mes recherches ont été publiées dans le livre : Industrial Enlightenment: science, technology and culture in Birmingham and the West Midlands 1760-1820, Manchester, Manchester University Press, 2008.

\footnotetext{
* Cet article est issu d'une communication faite au XIle Congrès international des Lumières - Sciences, Techniques et Cultures,
} Montpellier, 8-15 juillet 2007, dans la cadre de la table-ronde « Les techniques et les sciences au regard du bien public ». 
détaillée de ses activités autorisée par ces collections. Les Archives de Soho offrent un éclairage sur une époque, et la vue que nous avons n'est nullement confinée aux paysages rapidement urbanisés et industrialisés des Midlands. Birmingham, comme je l'expliquerai, est devenu dans la deuxième moitié du XVIIII siècle l'un des points nodaux des circuits de sociabilité scientifique en Europe.

I

Les Archives de Soho sont le nom donné à tous les fonds de l'entreprise et la correspondance de Matthew Boulton et de ses associés. Le nom dérive du lieu-dit de "Soho », à trois kilomètres de la ville de Birmingham, devenu le site du principal établissement manufacturier de Boulton. Précédemment, ces documents étaient connus comme les « Boulton \& Watt Papers ॥, une appellation qui avait le mérite d'attirer l'attention sur l'associé le plus ancien de Boulton et sur la principale source de notoriété de l'entreprise de l'historiographie des sciences et des techniques - James Watt, le pionnier de la vapeur. Cependant, la fabrication de machines à vapeur n'est qu'une dimension des activités de Boulton, et ce ne fut jamais le ressort premier de ses ambitions ni de ses préoccupations en tant que propagateur des valeurs des Lumières. Bien qu'il soit encore susceptible de causer la confusion parmi des chercheurs, l'intitulé moins réducteur d' "Archives de Soho ॥ couvre bien mieux la gamme des activités dans la manufacture de Soho. Il exprime également un peu mieux la présentation courante des fonds, comme nous le verrons.

Qui était donc Matthew Boulton ? Après tout, il figure plutôt rarement dans les index et les bibliographies des études universitaires sur le XVIII siècle. Dit d'une manière prosaïque, Matthew Boulton est un fabricant de boutons et de boucles de Birmingham -- comme son père avant lui. La famille avait des ateliers conséquents au centre de Birmingham pendant la première moitié du siècle, et les Boulton comptaient parmi le petit groupe d'entrepreneurs et d'innovateurs de Birmingham qui menaient l'évolution de la production, de la ferronnerie domestique et utilitaire aux articles de métal fantaisie connus alors sous le nom de " toys ». Après s'être marié avantageusement, Boulton ouvre en 1765 une manufacture construite dans le style néo-Palladien à la mode, à Soho, dans la périphérie de Birmingham. A son apogée, Soho est considéré comme le plus grand établissement manufacturier du monde occidental. II y développe une gamme de biens de consommation "populuxe " et se lance dans la fabrication d'articles de mode pour l'élite qui vont devenir des signes de distinction dans l'intelligentsia européenne de la fin des Lumières : vases néo-classiques, ormoulu, vaisselle d'argent et plaquée, urnes de thé, reproductions de peintures, verre taillé, médaillons et ainsi de suite (fig. 1).

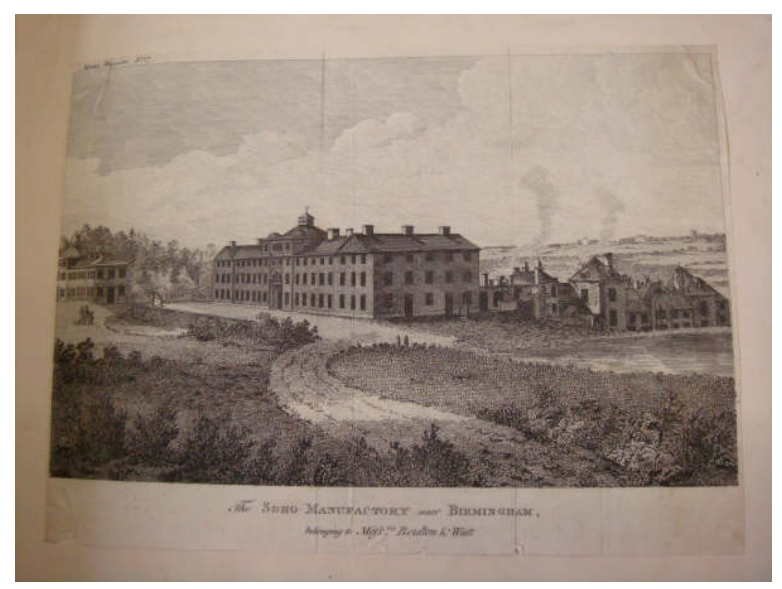

Figure 1 - La Manufacture de Soho, 1798, gravée par J. Walker (Birmingham City Archives, Timmins Collection)

En premier lieu, les Archives de Soho sont liées à ces activités - les marchés de commercialisation des articles et les clients concernés, à la fois en Angleterre et à l'étranger. Dans les années 1780, entre un quart et un tiers de la production des ateliers de Birmingham est exporté (en Europe et aux Amériques), et Boulton figure parmi les exportateurs les plus importants et les plus couronnés de succès. Ces archives d'entreprise sont considérables. De plus, si nous ajoutons l'entreprise de machines à vapeur qui commence à rapporter des dividendes dans les années 1780 et les affaires de monnayage (frappe de monnaie industrielle) qui sont bénéficiaires à la fin des années 1790, il s'agit d'un ensemble d'archives manuscrites qui exige un dépôt à part pour les emmagasiner. Les premières archives de l'entreprise actuellement conservées remontent aux années 1760 et les fonds les plus riches continuent jusqu'aux années 1840 , bien que le dépôt contienne également des matériaux jusqu'aux années 1890. On peut trouver dans les Archives de Soho environ 600 livres de comptes et de commandes, 1500 portefeuilles de moteurs et de machines, plusieurs douzaines de journaux intimes et de cahiers, environ 50000 feuilles volantes manuscrites et une quantité considérable de papiers de famille sur plusieurs générations.

Plus important encore dans l'immédiat, les Archives de Soho contiennent une substantielle correspondance - peut-être jusqu'à 250000 articles au total. A partir de 1780 environ, existent de nombreuses 
copies de lettres envoyées, en plus de celles reçues, parce que l'associé de Boulton, James Watt, est l'inventeur d'une presse à copier les lettres qui permet aux associés de faire des fac-similés durables et lisibles de leur correspondance. Ces «lettres de copie» (" copy letters ») sont collées dans les registres de correspondance ("letter books ») dans un ordre chronologique et couvrent non seulement leurs affaires, mais aussi une production épistolaire à caractère familial et également savant (« philosophique »). L'histoire de cet énorme fonds est plutôt compliquée. II suffit de dire que les papiers sont entrés en possession de la bibliothèque centrale de Birmingham à différents intervalles entre 1911 et 1994. La dernière grande acquisition a consisté en papiers de famille de Watt, dont une partie avait été déposée avec ceux de Boulton dès le milieu du XIXe siècle. En 1994, la partie restante de ces papiers de Watt est entrée dans le domaine public en raison d'une vente privée. Le conseil municipal de Birmingham a levé un million de livres pour leur achat, ce qui représentait à ce moment la plus forte somme jamais payée par une autorité locale pour une collection de manuscrits.

A peu près 90000 de ces lettres envoyées et reçues se rapportent à la période 1780 - 1820. Cependant, je n'ai étudié environ que 21000 des articles en question, pour la période allant de 1765 à 1820. Mon choix s'est concentré sur le contenu savant, c'est-à-dire sur la production de ces correspondants qui échangent des vues concernant la science expérimentale (philosophie naturelle) et son application à la technique. Pour les raisons qui s'éclairciront ci-dessous, ces deux thèmes avaient pour moi de fortes implications. Néanmoins, quand j'ai commencé ma recherche, l'interpénétration et le chevauchement des questions personnelles, des affaires et de la science furent une surprise. Pourtant, après avoir consulté environ 13000 lettres destinées à Matthew Boulton ou écrites par lui, et environ 8000 liées à James Watt, je n'ai plus eu aucun doute quant à la nature subjective, et même intime, de cette écriture épistolaire dans la deuxième moitié du XVIII' siècle ${ }^{4}$.

S'ajoutant à ce corpus de lettres de Boulton et Watt, existent aussi des correspondances très intéressantes avec Josiah Wedgwood, l'entrepreneur de poterie (environ 300 lettres), avec les médecins Erasmus Darwin (450 lettres) et William Withering (200 lettres), avec Richard Lovell Edgeworth et sa fille Maria (2 000 lettres) et avec Joseph Priestley, ministre du culte unitarien et représentant de la " philosophie naturelle »

4 Sur ces liens, voir Benoît Melançon éd., Penser par lettre, actes du colloque d'Azay-le-Ferron (mai 1997), Saint-Laurent, Québec, Fides, 1998. qui réside à Birmingham au long des années 1780 (environ 400 lettres). Une fois additionnés à la correspondance de Boulton et de Watt, ces fonds constituent un ensemble conséquent. Pour comparaison, il faut se rappeler que nous connaissons environ 17000 lettres reçues par Jean-Henri-Samuel Formey en sa qualité de secrétaire perpétuel de l'Académie royale des sciences de Prusse, un nombre équivalent pour Albrecht von Haller, 5500 pour Linné, 3000 pour l'essayiste et savant suisse Marc-Auguste Pictet, et peut-être 6000 pour Sir Joseph Banks - longtemps président de la Royal Society de Londres. Joseph Banks est I'un des grands épistoliers de l'époque et une édition exhaustive de sa correspondance vient seulement d'être éditée, ce qui rend peut-être ce chiffre caduc 5 .

La qualité, et non seulement la quantité de la correspondance concernant les transferts de savoirs pendant la deuxième moitié du XVIII siècle doit aussi être soulignée. Dans les seules Archives de Soho, existent des séries importantes de lettres émanant d'hommes de science comme Jean-André Deluc, Joseph Black, James Hutton, Ami Argand, Franz-Xaver Swediaur, du savant portugais connu sous le nom de Jean-Hyacinthe Magellan et de Rudolf Erich Raspe, auteur renommé des voyages du baron de Münchhausen. Pour cette raison, c'est sans exagération que l'on peut affirmer que les Archives de Soho ouvrent une fenêtre sur les Lumières scientifiques de la deuxième moitié du XVIII siècle. Le corpus offre aussi un aperçu précieux sur la fin du siècle des Lumières, c'est-à-dire sur la paralysie et la transformation radicale de la République des Lettres en Europe lors du bouleversement de la Révolution en France et des guerres napoléoniennes.

En plus des dossiers de correspondance mentionnés ci-dessus, mes recherches ont été nourries d'une autre source : les récits de voyage. La vogue

5 Sur la partie publiée de cette correspondance, voir Antoine-Claude Briasson et Nicolas-Charles-Joseph éd., Correspondance passive de Formey: lettres adressées à Jean-Henri-Samuel Formey, 1739-1770, Paris, Champion, 1996 ; Desmond King-Hele éd., The collected letters of Erasmus Darwin, Cambridge, Cambridge University Press, 2007 ; Richard Lowell Edgeworth et Maria Edgeworth, Memoirs of Richard Lovell Edgeworth, Esq. Begun by himself and his daughter Maria Edgeworth, Londres, Richard Bentley, 1844 ; Augustus J. C. Hare, The life and letters of Maria Edgeworth, 2 vols, Londres, Edward Arnold, 1894 ; Christina Colvin éd., Letters from England, 1813-1844, Oxford, Clarendon Press, 1971 ; John Towill Rutt, Life and correspondence of Joseph Priestley, 2 vols, Londres, Hunter and Eaton, 18311832 ; Marc-Auguste Pictet, 1752 - 1825. Correspondance : sciences et techniques, 3 vol., Genève, Slatkine, 1996-2000 ; http://linnaeus.c18.net/Doc/index.php. 


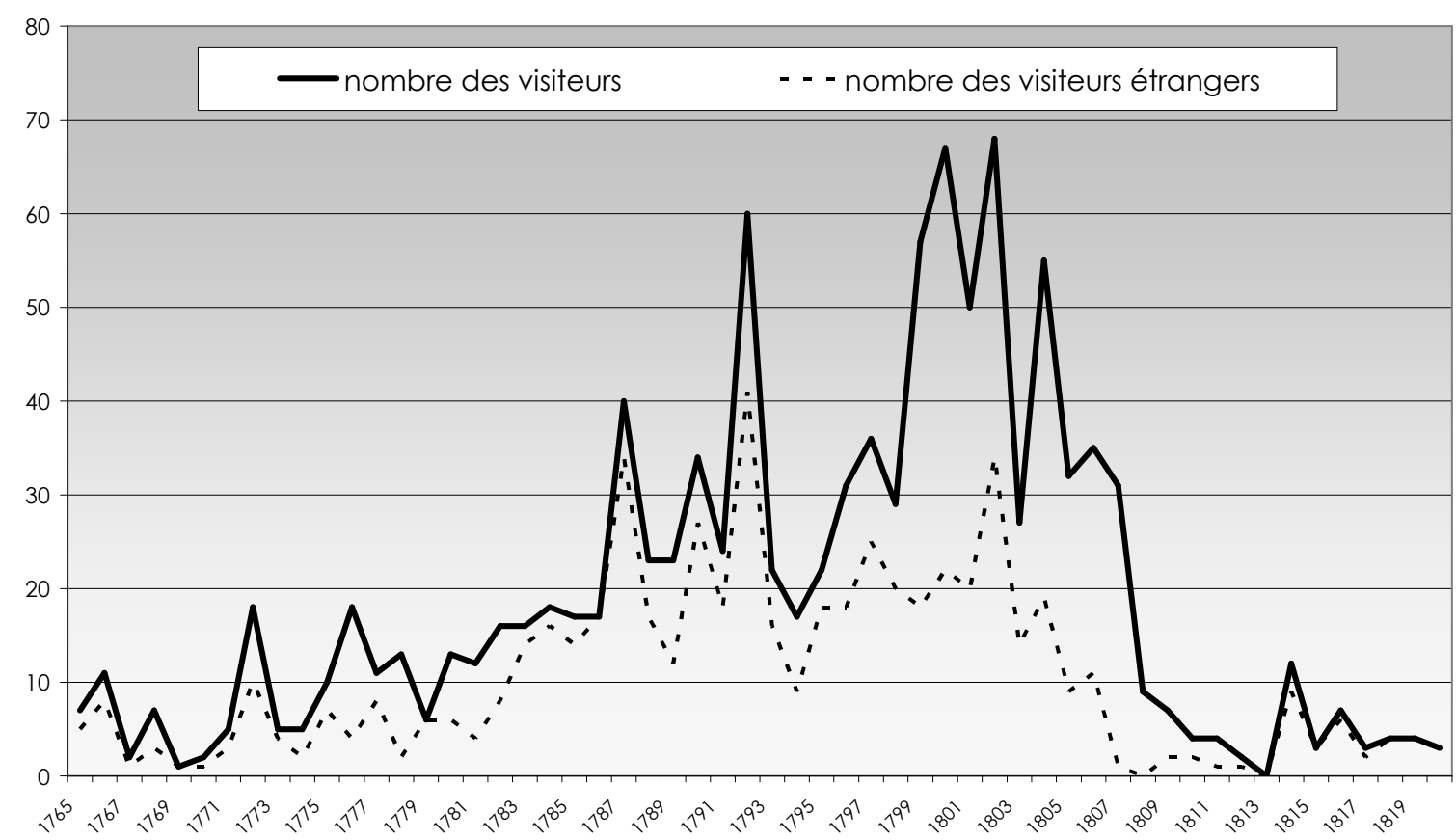

Figure 2 - Flux de visiteurs enregistrés à la Manufacture de Soho, 1765-1820 (Source : Birmingham Central Library MS 3147; MS 3219; MS 3782)

des voyages et le marché pour cette littérature après 1760 n'ont pas besoin d'être soulignés. Seulement une poignée de journaux intimes de voyage anglais ont survécu pour le XVIIe siècle, et pas plus de vingt environ pour la première moitié du XVIII ${ }^{\mathrm{e}}$ siècle. Mais après le milieu du siècle, c'est une histoire différente. Environ 140 récits peuvent être identifiés entre 1750 et 1800, et 270 entre 1800 et $1850^{6}$. Beaucoup sont des traductions éditées de journaux allemands et français, naturellement - ce qui les rend d'autant plus intéressants. On a également la preuve d'une évolution nette dans l'objectif et le cadre mental qui se mettent en place alors que la description technologique devient un enjeu spécifique des voyages, en particulier chez les visiteurs scandinaves et prussiens en Grande-Bretagne. Une certaine idée des flux de ce " tourisme industriel », et des origines des milliers de voyageurs qui ont effectué une visite à la manufacture de Soho de Matthew Boulton est proposée dans les figures 2 et 3.

6 See Robin Gard éd., The observant traveller: diaries of travel in England, Wales and Scotland in the County Record Offices of England and Wales, Londres, HMSO, 1989 ; aussi George Edwin Fussell, The exploration of England : a select bibliography of travel and topography, 1570 - 1815, Londres, Mitre Press, 1935.
$\|$

Comment ai-je étudié les Archives de Soho ? Au commencement, mes enquêtes n'avaient d'autre but que d'explorer la dimension internationale de l'activité scientifique, technologique et entrepreneuriale à Birmingham et dans les West Midlands pendant la deuxième moitié du XVIII siècle. II faudrait dire que beaucoup de chercheurs ont précédemment ouvert la voie dans ces archives - principalement en vue d'étudier les techniques relatives à la vapeur. En général, ce qu'ils découvraient sur le contexte européen plus large de l'activité technologique était délaissé, car jugé comme inutile. J'ai commencé mes enquêtes en 1996, m'intéressant aux papiers de la famille de James Watt nouvellement acquis, mais je me suis bientôt rendu compte que Matthew Boulton était l'associé le plus intéressant. J'ai également conclu que la meilleure manière d'explorer l'économie de la connaissance, l'évolution technologique et l'esprit d'entreprise au XVIIII siècle était de se concentrer sur les fonds de correspondance décrits plus haut. La littérature épistolaire et de voyage tend à être négligée par les historiens de la culture scientifique : or il est passionnant de constater que dans ce domaine toutes les routes ont croisé Birmingham. Néanmoins un problème demeurait, et il est un de ceux que rencontre tout his- 


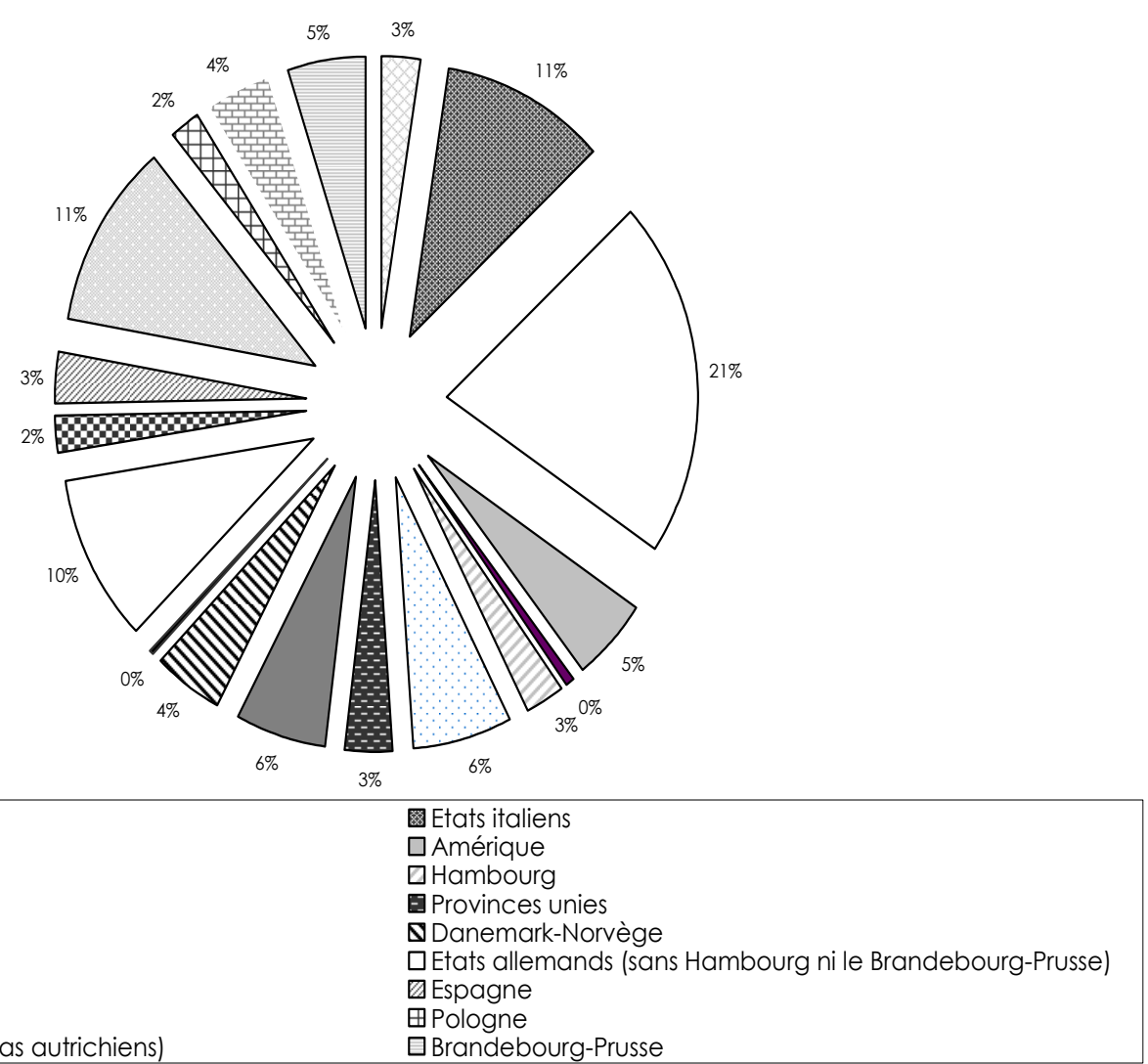

Figure 3-Visiteurs étrangers de la Manufacture de Soho, 1765- 1820

(Source : Birmingham Central Library MS 3147; MS 3219; MS 3782)

\begin{tabular}{|l|r|r|}
\hline Inconnue & $\mathbf{1 5}$ & $2,60 \%$ \\
\hline États italiens & $\mathbf{6 2}$ & $10,60 \%$ \\
\hline France & $\mathbf{1 2 4}$ & $21,20 \%$ \\
\hline Amérique & $\mathbf{3 0}$ & $5,10 \%$ \\
\hline Canada & $\mathbf{2}$ & $0,30 \%$ \\
\hline Hambourg & $\mathbf{1 6}$ & $2,70 \%$ \\
\hline Cantons suisses & $\mathbf{3 7}$ & $6,30 \%$ \\
\hline Provinces unies & $\mathbf{1 8}$ & $3,10 \%$ \\
\hline Suède (et Finlande) & $\mathbf{3 4}$ & $5,80 \%$ \\
\hline Danemark-Norvège & $\mathbf{2 5}$ & $4,30 \%$ \\
\hline Empire ottoman & $\mathbf{2}$ & $0,30 \%$ \\
\hline États allemands (sans Hambourg ni le Brandebourg-Prusse) & $\mathbf{5 9}$ & $10,10 \%$ \\
\hline Portugal & $\mathbf{1 2}$ & $2,10 \%$ \\
\hline Espagne & $\mathbf{1 9}$ & $3,30 \%$ \\
\hline Russie & $\mathbf{6 4}$ & $11 \%$ \\
\hline Pologne & $\mathbf{1 1}$ & $1,90 \%$ \\
\hline Empire Habsbourg (et Pays-bas autrichiens) & $\mathbf{2 4}$ & $4,10 \%$ \\
\hline Brandebourg-Prusse & $\mathbf{3 0}$ & $5,10 \%$ \\
\hline
\end{tabular}


torien des réseaux de correspondance : comment traiter ce type de sources afin de les transformer en un matériau pour la recherche?

Dans cet effort, j'ai été considérablement aidé par le travail de chercheurs comme Margaret C. Jacob, Larry Stewart et l'économiste et historien Joel Mokyr. Le concept de «Lumières industrielles » (« industrial Enlightenment ») que Mokyr semble avoir été le premier à expliciter, me semble une aide puissante dans la compréhension de ce qui s'est produit à Birmingham, à Manchester ou à Leeds pendant la deuxième moitié du XVIIII siècle et les premières décennies du XIXe siècle. Mais comme l'exprime Mokyr dans son livre The gifts of Athena : historical origins of the knowledge economy (2002), les « Lumières industrielles " sont surtout un constat théorique. Avec des archives du calibre de celles de Soho, j'en suis venu à l'idée qu'il serait intéressant de mettre à l'épreuve ces « Lumières industrielles ॥ situées dans l'intervalle entre la révolution scientifique et la révolution industrielle. Et, si cette notion passait l'épreuve, il fallait lui donner de la chair et du sang. Cependant, mes enquêtes se sont prolongées au-delà de celles de Mokyr à certains égards puisqu'elles embrassent la question de la culture scientifique en soi et qu'elles incluent une enquête sur le rôle de la religion dans la propagation de la science et de l'industrie du XVIII siècle. Néanmoins, le motif central de mon travail pendant ces dernières années a été d'interroger les arguments d'abord formulés par Joel Mokyr.

Cette recherche est maintenant complète et a été éditée dans une monographie en 20087. Le livre n'est pas une biographie de Matthew Boulton, ni même un exposé complet de l'histoire de la manufacture de Soho. Mais sa sortie a été prévue pour coïncider avec le bicentenaire de la mort de Boulton. S'il n'est pas une biographie, le livre complète donc de manière significative notre compréhension de l'individu et de ses efforts de créer une voie anglaise et provinciale des Lumières européennes. Quelles sont les conclusions du livre ? Pour éviter de rallonger cet article, je les récapitulerai comme suit.

- Au cours du mon enquête, le scénario des "Lumières industrielles » de Joel Mokyr - au départ, une hypothèse intéressante - est devenu convaincant. Néanmoins, j' attirerai l'attention sur trois secteurs pour lesquels cette explication semble exiger davantage de réflexion. En faisant resurgir le débat sur le rôle des savoirs dans l'évolution technologique, Mokyr ne semble pas ancrer solidement la distinction entre

7 Voir note 3 ci-dessus. le savoir propositionnel (« knowledge what ») et celui prescriptif («knowledge for »). D'ailleurs, je doute qu'il tienne compte suffisamment des effets des « cultures" de la connaissance - spécifiquement pour autant qu'elles ont gêné la diffusion de la connaissance. Ma troisième prise de position est moins une critique qu'une exhortation. Les échanges entre les savants et les fabricants sont le processus clé des « Lumières industrielles ॥, mais cet interface est susceptible d'une définition beaucoup plus fine que celle de Mokyr dans The gifts of Athena ${ }^{8}$. L'accumulation de preuves (et non des moindres, le cas de Boulton et de la manufacture de Soho) démontre que les fabricants et les savants en Angleterre et en Écosse au XVIII siècle recouvrent souvent les mêmes personnes - facilitant de ce fait, le processus de production et d'application des savoirs pratiques?.

- Il est possible d'identifier et d'analyser dans des contextes régionaux particuliers une voie spécifique des Lumières en Angleterre. À Birmingham et dans les villes environnantes, les Lumières sont un phénomène propre à la classe moyenne et aux artisans de haut niveau. Je soulignerais, aussi, que cette tendance est étroitement liée aux courants intellectuels venus de l'Europe continentale. Si les Dissenters sont particulièrement dévoués aux Lumières dans leur expression locale, à Birmingham, le phénomène n'est en rien une création exclusivement non-conformiste. Quant à la Lunar Society, je ne partage pas l'interprétation qui a longtemps prévalu depuis les recherches de Robert E. Schofield sur l'importance de ce milieu' ${ }^{10}$, et que Joel Mokyr reprend à son compte" ${ }^{11}$ Selon cette lecture, la Lunar Society constitue le foyer technologique de la révolution industrielle. Au contraire, je considère celle-ci avant tout comme l'émanation de la culture associative propre aux Lumières.

8 Joel Mokyr reconsidère cette question dans un article, « The intellectual origins of modern economic growth $»$, Journal of economic history, 65, 2005, pp. 285-343. Voir aussi Liliane Hilaire-Pérez, « Technology as public culture in the eighteenth century : the artisan's legacy $"$, History of science, 45, 2007, pp. 135-153.

9 Sur ce processus, voir Margaret C. Jacob et Larry Stewart, Practical matter: Newton's science in the service of industry and empire, 1687-1814, Cambridge, Mass., Harvard University Press, 2004, pp. 127-138.

10 Robert E. Schofield, The Lunar Society of Birmingham : a social history of provincial science and industry in eighteenthcentury England, Oxford, Clarendon Press, 1957.

11 Joel Mokyr, The gifts of Athena : historical origins of the knowledge economy, Princeton, NJ, Princeton University Press, 2002, p. 44, note 17. 
- La sociabilité scientifique qui se manifeste dans les réseaux épistolaires des philosophes naturels a pu être un vecteur particulièrement efficace et rapide de la science en Europe pendant les décennies courant de la fin de la Guerre de Sept Ans à l'éclatement de la Révolution française (1763 - 1789). L'un des exemples que je souligne dans mon livre est l'effort collectif pour élucider la composition chimique de l'eau'2. Mais il est certain que certaines formes de savoir ont circulé mieux que d'autres et à différents rythmes selon les milieux sociaux et professionnels concernés. D'ailleurs, comme en font l'expérience les savants itinérants tel qu'Alessandro Volta ${ }^{13}$, la République des Lettres au XVIII siècle n'est pas un espace homogène. Elle comporte de nombreux sous-ensembles, croisements et points nodaux.

- Malgré l'extension géographique et l'intensité des échanges de la science expérimentale en Europe pendant la deuxième moitié du XVIII siècle, la production et l'adoption des savoirs pratiques sont restées dépendantes de facteurs culturels et institutionnels qui n'ont pas été facilement surmontés. La connaissance tacite, ou plutôt le " savoir-faire " n'a pas bien transité, et la forte diversité des cultures scientifiques des différents pays européens ne pouvait pas être facilement réduite. Dans mon étude, j'utilise une approche comparative pour illustrer ce fait, en prenant comme exemple la diffusion des techniques de la vapeur en Angleterre, en France, en Hollande, en Prusse et en Scandinavie. Le cas hollandais est particulièrement éloquent sur la façon dont les contraintes culturelles et politiques peuvent interférer pour freiner le processus de diffusion ${ }^{14}$.

- La science et la technique ont-elles été liées de manière causale au XVIII siècle ? J'en suis persuadé, à la lumière de l'analyse précise des activités industrielles de James Watt, de Matthew Boulton et d'un certain nombre de fabricants qui leur sont proches. Des hommes tels que Watt, Boulton, Wedgwood et le chimiste et industriel James Keir ne sont que la pointe visible

12 Peter M. Jones, Industrial enlightenment, op. cit. note 3, pp. 106-109.

13 Giuliano Pancaldi, Volta : science and culture in the age of Enlightenment, Princeton, Princeton University Press, 2003, pp. 104, 151, 169-176.

14 P. M. Jones, Industrial Enlightenment, op. cit. note 3, pp. 146-49; Jan Adrianus Verbruggen éd., The Correspondence of Jan Daniël Huichelbos van Liender (1732-1809) with James Watt (1736-1819) and Boulton \& Watt, supplemented by a few related Documents compiled and annotated by Jan $A$. Verbruggen, impression privée, 2005, pp. 26-28, 201. d'une espèce identifiable de savants-fabricants opérant au cœur des « Lumières industrielles » en GrandeBretagne. L'extension de cette culture du savoir appliqué est très difficile à mesurer, mais il est sûr qu'elle s'est déployée au-delà des partenaires de l'entreprise, jusqu' aux principaux employés (ingénieurs, contremaîtres, constructeurs de machines, etc.) de la manufacture de Soho. En effet, dans plusieurs cas, j'ai pu montrer des liaisons directes entre la théorie scientifique et la pratique industrielle - comme d'autres chercheurs l'ont fait de manière approfondie pour Manchester et Leeds's.

- Cette provesse technologique peut-elle être liée de manière causale aux impératifs, ou peut-être aux caractères culturels, de la religion Dissent ? Après avoir pesé les arguments de Max Weber et de Robert K. Merton, j'ai tiré la conclusion qu'aucune relation de ce type ne peut être fermement établie ${ }^{16}$. James Watt est presbytérien, mais Matthew Boulton est anglican, de même que James Keir et Samuel Garbett ; il n'est donc pas possible d'expliquer l'essor technique et innovant des industriels et des petits entrepreneurs de Birmingham en termes si schématiques. Seulement dans le cas des Quakers, l'éthique s'est fondée sur la conduite des affaires - mais une éthique tenant davantage aux ressources culturelles de cette secte qu'à ses croyances religieuses ${ }^{17}$.

- La conclusion qui émerge de mon livre concerne le remodelage de la République des Lettres à la fin du siècle. En dépit d'un argument persistant dans l'historiographie des sciences selon lequel «les sciences n'aient jamais été en guerre "18, j' ai montré que ce constat était infirmé par la Révolution française et les guerres napoléoniennes. En 1795, James Watt accuse les révolutionnaires français d'avoir « assassiné la philo-

15 P. M. Jones, Industrial Enlightenment, op. cit. note 12, pp. 126-128; M. C. Jacob et L. Stewart, Practical patter, op. cit., pp. 127-138.

16 Voir Peter M. Jones, « Minorities and the culture of science and technology. A case study of Birmingham Dissenters 1760-1820 11, Documents pour l'histoire des techniques, 15, 2008, pp. 58-63.

17 P. M. Jones, Industrial Enlightenment, op. cit. note 12, pp. 181-4, 187-188, 199 ; aussi Geoffrey Cantor, Quakers, Jews, and science : religious responses to modernity and the sciences in Britain, 1650-1900, Oxford, Oxford University Press, 2005.

18 Cette remarque est attribuée au médecin pionnier de l'inoculation, Edward Jenner : voir A. Hunter Dupree, " Nationalism and Science: Sir Joseph Banks and the Wars with France ", dans David Henry Pinkney et Theodore Ropp éd., A festschrift for Frederick B. Artz, Durham, Duke University Press, 1964, p. 37. 


\section{Matthew Boulton et ses réseaux}

sophie $1{ }^{19}$ - il se réfère à la suppression de l'Académie des sciences en 1793 et à l'exécution du chimiste Antoine-Laurent Lavoisier l'année suivante. Autant que l'on puisse en juger d'après les fonds de la correspondance dans les Archives de Soho, la soi-disant République des Lettres est déjà sévèrement altérée en 1795. Elle se rétablit légèrement pendant l'intermède de la Paix d'Amiens (1801 - 1803), mais cesse presque totalement de fonctionner suite aux prises d'otages de mai 1803 dirigées contre les sujets britanniques et aux blocus de 1806 - 1807. Une des conséquences de cette rupture est le défilé d'Européens en quête d'information technique dans ville de Birmingham une fois que la paix est restaurée en 1814 - 1815 et l'essor renouvelé des voyages européens.

\section{Conclusion}

Entre 2000 et 2005, les Archives de Soho ont été entièrement re-cataloguées, de manière experte. En conséquence, il est maintenant plus facile de se servir de cette richesse documentaire. Grâce à l'accès aux inventaires modernes et aux outils de recherche, les universitaires peuvent apprécier le potentiel des archives et l'exploiter pleinement. Si l'on met de côté le thème particulier de cet article, d'autres

19 Birmingham Central Library MS 3219/4/124 J. Watt snr to W. Roebuck, Birmingham, 19 August 1795. sujets très attractifs attendent les chercheurs et les étudiants intéressés par la culture matérielle : consommation et publicité ; groupes de pression industriels ; art et iconographie industriels; histoire des entreprises et commercialisation; histoire du travail ; brevets et inventions; codification de l'écriture épistolaire ; voyages; numismatique et monnayage.

En 2009, année du bicentenaire de la mort de Matthew Boulton, bien des signes suggèrent que les chercheurs commencent à s'inscrire dans ces problématiques. L'étude systématique du rôle de Boulton dans le développement des techniques du métal et la mécanisation de la production du monnayage (les frappes de Soho) a commencé en 2006, de même la recherche iconographique sur les divers sites industriels liés au patronyme de Boulton \& Watt. Ce renouvellement d'intérêt a assurément été stimulé par les expositions publiques de l'année du bicentenaire, et par le colloque tenu à l'université de Birmingham qui a convié des chercheurs du monde entier pour discuter le procès par lequel « la nation de Newton et de Locke est devenue la nation de Boulton et Watt $"{ }^{20}$.

(Texte traduit de l'anglais par Liliane Pérez)

20 Paul Langford, Englishness identified: manners and character, 1650-1850, Oxford, Oxford University Press, 1992, p. 76. 